\title{
MEDIOS, TÉCNICAS E INSTRUMENTOS DE EVALUACIÓN FORMATIVA EN ZOOLOGÍA (GRADO DE BIOLOGÍA): UNA VISIÓN DE CONJUNTO
}

Means, techniques and instruments of formative asseessment in Zoology (degree of Biology): an overview

Meios, técnicas e métodos de avaliação formativa na Zoologia (grado em Biologia): uma visão geral

Andrés Arias (1)

Irene Fernández-Rodríguez (2)

Miguel González-Menéndez (3)

Nuria Anadón (4)

(1) Departamento de Biología de Organismos y Sistemas (Zoología), Universidad de Oviedo, España. Teléfono: +34 985104822. Correo electrónico: ariasandres.uo@uniovi.es / andresarias.rguez@gmail.com

(2) Departamento de Biología de Organismos y Sistemas (Zoología), Universidad de Oviedo, España. Correo electrónico: irefdezrguez@hotmail.com / u223866@uniovi.es

(3) The English School of Asturias, Pruvia de Abajo, España. m.gonzalez@colegioingles.co.uk

(4) Departamento de Biología de Organismos y Sistemas (Zoología), Universidad de Oviedo, España. Correo electrónico: nanadon@uniovi.es

\section{Resumen}

En este trabajo se presenta una revisión de los medios, técnicas e instrumentos de evaluación formativa del aprendizaje en la asignatura de Zoología en los distintos grados de Biología de las universidades españolas. Los medios de evaluación más utilizados en nuestras universidades son los de tipo escrito, encabezados por el examen y seguidos por el trabajo escrito, la presentación oral y los controles. Las técnicas de evaluación predominantes son las aplicadas unilateralmente por el profesorado sin que el alumnado participe en el proceso evaluativo. En base a la revisión de las guías docentes no se pueden analizar objetivamente los instrumentos utilizados en la Medios, técnicas e instrumentos de evaluación formativa en Zoología (Grado de Biología): una visión de conjunto 
evaluación, por lo que consideramos que este aspecto, junto con una mayor claridad a la hora de explicitar los medios y las técnicas de evaluación utilizados, debería ser tenido en cuenta en la elaboración de los planes docentes de la asignatura.

Palabras clave: Evaluación; calidad educativa; educación superior; Ciencias Naturales; Universidad

\begin{abstract}
We are presenting a review of the means, techniques and instruments of formative assessment of learning in the subject of Zoology in the degree of Biology of Spanish universities. In the studied universities the most used means of assessment are the written-type, headed by the exam and followed by the assignments, the oral presentation and the tests. The predominant evaluation techniques are those unilaterally applied by the teacher without intervention of the students in the evaluative process. Based on the revision of the teaching guides, it is not possible to analyze the instruments used in the evaluation, so we consider that this aspect should be taken into account in the preparation of the teaching plans. We also suggest more clarity in drafting of the means and the techniques of evaluation of teaching guides.
\end{abstract}

Keywords: Assessment; educational quality; higher education; Natural Sciences; University

\title{
Resumo
}

Este trabalho apresenta uma revisão dos meios, técnicas e ferramentas de avaliação formativa da aprendizagem na disciplina de Zoologia nos diferentes graus de Biologia das universidades espanholas. Os meios de avaliação mais utilizados em nossas universidades são os escritos, liderada pelo exame e seguido por trabalho escrito, a apresentação oral e controles. As técnicas de avaliação vigentes são aplicadas unilateralmente por professores sem alunos participam no processo de avaliação. Com base na revisão dos guias de ensino não pode ser objetivamente analisar os instrumentos utilizados na avaliação, e acreditamos que isso deve ser levado em conta na preparação de planos de ensino, Também consideramos oportuno maior clareza em usando a mídia e técnicas de avaliação em às diferentes universidades.

Palavras-chave: Avaliação; qualidade da educação; ensino superior; Ciências Naturais; Universidade

Medios, técnicas e instrumentos de evaluación formativa en Zoología (Grado de Biología): una visión de conjunto 


\section{Introducción}

Durante los últimos años las universidades españolas han experimentado un gran proceso de reestructuración para adaptarse al Espacio Europeo de Educación Superior (EEES) y al sistema de transferencia y acumulación de créditos (ECTS). Una de la novedades más representativas de este proceso de consolidación hacia el EEES ha sido la inclusión de la evaluación formativa en los nuevos grados universitarios y al mismo tiempo, esto ha supuesto uno de sus mayores retos (Palomares-Ruiz, 2011; Hamodi et al., 2015). La evaluación formativa, un concepto originalmente desarrollado por Scriven (1967), es actualmente entendido como “todo proceso de constatación, valoración y toma de decisiones cuya finalidad es optimizar el proceso de enseñanza-aprendizaje desde una perspectiva humanizadora y no como un mero fin calificador” (Hamodi et al., 2015).

El grado en Biología se imparte actualmente en 28 universidades españolas tanto públicas como privadas y dentro de éste, la asignatura de Zoología es una materia troncal. La Zoología es una ciencia biológica básica y fundamental. Su conocimiento permite el acceso a otras disciplinas biológicas que tratan aspectos más específicos relacionados con los animales (e.g. fisiología, ecología, genética).

El principal objetivo de este trabajo es realizar una comparación objetiva de los distintos medios, técnicas e instrumentos utilizados en la evaluación de la asignatura de Zoología del grado de Biología en las universidades españolas. Dicha comparación nos permitirá obtener una visión general sobre la adaptación de la Zoología a los procesos de convergencia europea, así como diferenciar patrones generales y específicos en la utilización de los diferentes medios, técnicas e instrumentos de evaluación.

\section{Método}

Se han revisado los planes o guías docentes relativos a la asignatura de Zoología de los distintos grados de Biología de las 28 universidades españolas que lo ofertan, prestando especial atención a los medios, técnicas e instrumentos de evaluación formativa recogidos en éstos. La terminología utilizada para clasificación de dichos medios, técnicas e instrumentos ha sido la propuesta por Hamodi et al. (2015). Dichos autores subdividen los medios en “escritos”, “orales” y “prácticos”, asimismo diferencian dos 
tipos de técnicas: en las que interviene el alumnado y en las que el alumnado no participa (Hamodi et al., 2015).

\section{Resultados y discusión}

La información obtenida de la revisión de los planes docentes de la asignatura de Zoología en relación con los medios de evaluación formativa se muestra en la Tabla 1.

\section{Tabla 1.}

Relación de los medios de evaluación de la asignatura de Zoología utilizados en las distintas universidades españolas

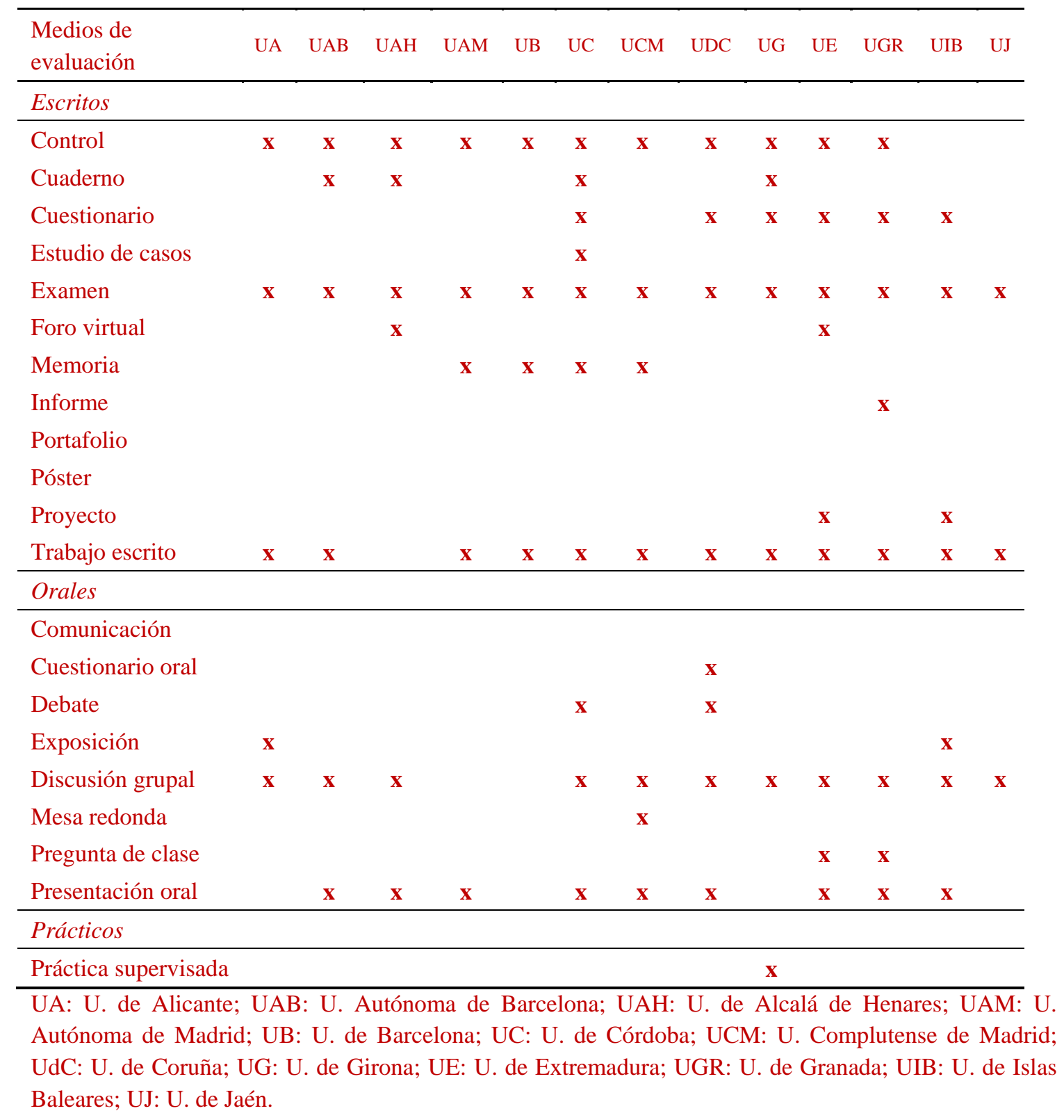

Medios, técnicas e instrumentos de evaluación formativa en Zoología (Grado de Biología): una visión de conjunto 
Tabla 1 (continuación)

\begin{tabular}{|c|c|c|c|c|c|c|c|c|c|c|c|c|c|c|c|}
\hline $\begin{array}{l}\text { Medios de } \\
\text { evaluación }\end{array}$ & UL & ULL & UM & UMA & UN & UO & UPF & UPV & URJ & US & USA & USC & uv & UVC & UVI \\
\hline \multicolumn{16}{|l|}{ Escritos } \\
\hline Control & & & & $\mathbf{x}$ & & & & & $\mathbf{x}$ & $\mathbf{x}$ & $\mathbf{x}$ & & $\mathbf{x}$ & & \\
\hline Cuaderno & & & $\mathbf{x}$ & & $\mathbf{x}$ & $\mathbf{x}$ & & $\mathbf{x}$ & & & & & & & \\
\hline Cuestionario & $\mathbf{x}$ & & $\mathbf{x}$ & & & & $\mathbf{x}$ & & & & $\mathbf{x}$ & & $\mathbf{x}$ & $\mathbf{x}$ & $\mathbf{x}$ \\
\hline $\begin{array}{l}\text { Estudio de } \\
\text { casos }\end{array}$ & & & & $\mathbf{x}$ & & & & $\mathbf{x}$ & & & & & & & $\mathbf{x}$ \\
\hline Examen & $\mathbf{x}$ & $\mathbf{x}$ & $\mathbf{x}$ & $\mathbf{x}$ & $\mathbf{x}$ & $\mathbf{x}$ & $\mathbf{x}$ & $\mathbf{x}$ & $\mathbf{x}$ & $\mathbf{x}$ & $\mathbf{x}$ & $\mathbf{x}$ & $\mathbf{x}$ & $\mathbf{x}$ & $\mathbf{x}$ \\
\hline Foro virtual & & & & & & & & & & & $\mathbf{x}$ & & & & \\
\hline Memoria & & & & & & & & & & & $\mathbf{x}$ & & $\mathbf{x}$ & & \\
\hline Informe & & $\mathbf{x}$ & $\mathbf{x}$ & & $\mathbf{x}$ & & & & & & & & & $\mathbf{x}$ & \\
\hline Portafolio & & & $\mathbf{x}$ & & & & & & & & & & & & \\
\hline Póster & & & & & & & & & & & & & $\mathbf{x}$ & & \\
\hline Proyecto & & & & & & & & $\mathbf{x}$ & & $\mathbf{x}$ & & & & & $\mathbf{x}$ \\
\hline Trabajo escrito & $\mathbf{x}$ & $\mathbf{x}$ & & $\mathbf{x}$ & $\mathbf{x}$ & $\mathbf{x}$ & & $\mathbf{x}$ & $\mathbf{x}$ & & $\mathbf{x}$ & $\mathbf{x}$ & $\mathbf{x}$ & $\mathbf{x}$ & $\mathbf{x}$ \\
\hline \multicolumn{16}{|l|}{ Orales } \\
\hline Comunicación & & & & & & & & & & & & & $\mathbf{x}$ & & \\
\hline $\begin{array}{l}\text { Cuestionario } \\
\text { oral }\end{array}$ & & & $\mathbf{x}$ & $\mathbf{x}$ & & & & & & & & & & & \\
\hline Debate & & & & & & & & & & & & & & & $\mathbf{x}$ \\
\hline \multicolumn{16}{|l|}{ Exposición } \\
\hline $\begin{array}{l}\text { Discusión } \\
\text { grupal }\end{array}$ & & & $\mathbf{x}$ & & $\mathbf{x}$ & $\mathbf{x}$ & & & & & $\mathbf{x}$ & & $\mathbf{x}$ & $\mathbf{x}$ & $\mathbf{x}$ \\
\hline Mesa redonda & & & & & & & $\mathbf{x}$ & & & $\mathbf{x}$ & & & & $\mathbf{x}$ & $\mathbf{x}$ \\
\hline $\begin{array}{l}\text { Pregunta de } \\
\text { clase }\end{array}$ & & & & $\mathbf{x}$ & & & & & & & & & & & \\
\hline $\begin{array}{l}\text { Presentación } \\
\text { oral }\end{array}$ & $\mathbf{x}$ & $\mathbf{x}$ & & & $\mathbf{x}$ & $\mathbf{x}$ & & $\mathbf{x}$ & $\mathbf{x}$ & & $\mathbf{x}$ & $\mathbf{x}$ & $\mathbf{x}$ & & \\
\hline \multicolumn{16}{|l|}{ Prácticos } \\
\hline \multicolumn{16}{|l|}{$\begin{array}{l}\text { Práctica } \\
\text { supervisada }\end{array}$} \\
\hline $\begin{array}{l}\text { UL: U. de León; } \\
\text { UO: U. de Oviec } \\
\text { de Sevilla; USA } \\
\text { de Vic-U. Centra }\end{array}$ & le & $\begin{array}{l}\text { La L } \\
\text { Pom } \\
\text { Ianca } \\
\text {; UV }\end{array}$ & $\begin{array}{l}\text { guna, } \\
\text { eu Fa } \\
\text { USC } \\
\text { I: U. }\end{array}$ & $\begin{array}{r}\text { UM: } \\
\text { bra; } \mathrm{L} \\
\mathrm{U} \\
\text { de } \mathrm{Vi}\end{array}$ & $\begin{array}{l}\text { U. d } \\
\text { PV: } \\
\text { de S } \\
0\end{array}$ & $\begin{array}{l}\mathrm{Mu} \\
\mathrm{U} . \\
\text { antic }\end{array}$ & $\begin{array}{l}\text { cia; } \\
\text { le } \mathrm{Pa} \\
\text { go de }\end{array}$ & $\begin{array}{l}\mathrm{JMA} \\
\text { is Va } \\
\text { Com }\end{array}$ & $\begin{array}{l}\text { U. de } \\
\text { co; U } \\
\text { postel }\end{array}$ & Má & $\begin{array}{l}\text { aga } \\
\text { U. Re } \\
\text { V: U. }\end{array}$ & $\begin{array}{l}\text { N: L } \\
\text { Juar } \\
\text { de } \mathrm{Va}\end{array}$ & $\begin{array}{l}\mathrm{d} \\
\mathrm{Car} \\
\text { enci }\end{array}$ & $\begin{array}{r}\mathrm{Na} \\
\text { os; } \mathrm{L} \\
\text {; UV U }\end{array}$ & $\begin{array}{l}\text { tarra; } \\
\text { S: U. } \\
\text { C: U. }\end{array}$ \\
\hline
\end{tabular}

De forma general los medios de evaluación escritos (con un total de 12) predominan sobre los orales (8) y los prácticos (1), estos últimos únicamente representados por la evaluación de una práctica supervisada en la Universidad de Girona (Tabla 1). Los medios de evaluación más utilizados (aprox. en el 60\% de las universidades) han sido el examen -teórico, práctico y/o de reconocimiento de visu- (en todas), el trabajo escrito (en 24 universidades), la presentación oral (en 18) y el control Medios, técnicas e instrumentos de evaluación formativa en Zoología (Grado de Biología): una visión de conjunto 
(en 16). Reseñablemente, sólo la universidad de Murcia utiliza el "portafolio" en la evaluación de la Zoología, dicho medio ha sido considerado de gran utilidad en los procesos de enseñanza-aprendizaje en educación superior, ya que permite que el estudiante se convierta en el organizador de su propio aprendizaje y es, además, especialmente indicado en el contexto del EEES (Palomares-Ruiz, 2011; Hamodi et al., 2015). Por tanto, consideramos que la generalización de su utilización en los grados de Biología constituiría una aportación significativa a la calidad de éstos.

En cuanto a las técnicas de evaluación de la Zoología, en más del 80\% de las universidades españolas el alumnado no interviene en el proceso evaluativo y la evaluación se basa en el análisis documental/de producciones y la observación del alumno/a o del grupo de alumnos/as. Por el contrario las técnicas en las que alumnado participa en el proceso de evaluación, únicamente han sido registradas en las Universidades de Alcalá y de las Islas Baleares, con utilización de la “autoevaluación” y la “evaluación entre iguales” y en la Universidad de Málaga, en la que se fomenta la “evaluación compartida o colaborativa” mediante entrevistas con el alumnado.

Finalmente, cabe destacar que los instrumentos de evaluación utilizados en las distintas universidades no han podido ser objetivamente analizados en base a la información recogida en los planes docentes, ya que ésta, o bien, no ha sido explicitada o resulta ser poco clara. Es muy probable que la mayoría de las universidades utilicen "listas de control” para evaluar asistencias a clases, prácticas y/o seminarios, pero estos instrumentos no aparecen reflejados en los planes de la asignatura.

\section{Conclusiones}

La revisión de las guías docentes de Zoología de las universidades españolas evidencia que, en la mayoría de los casos, la metodología de evaluación aparece mucho menos explicitada que la metodología de calificación e incluso en algunos planes se llega a confundir ambos conceptos. En Zoología, los medios de evaluación escritos predominan sobre los orales y los prácticos, siendo el examen el único medio utilizado en todas las universidades españolas, seguido, por orden de utilización, por el trabajo escrito, la presentación oral y los controles. Recomendamos la generalización del uso del portafolio como medio de evaluación escrito, dados los importantes beneficios que aporta al proceso de enseñanza-aprendizaje. Las técnicas de evaluación predominantes Medios, técnicas e instrumentos de evaluación formativa en Zoología (Grado de Biología): una visión de conjunto 
son las aplicadas unilateralmente por el profesorado sin que el alumnado participe en el proceso evaluativo. En base a las guías docentes no se pueden analizar objetivamente los instrumentos utilizados en la evaluación, aspecto que consideramos debería ser tenido en cuenta, junto con una mayor explicitación de los medios y técnicas de evaluación, a la hora de la elaboración de estas guías.

\section{Referencias}

Hamodi, C., López, V. M., \& López, A. T. (2015). Medios, técnicas e instrumentos de evaluación formativa y compartida en Educación Superior. Perfiles Educativos, $147,146-161$.

Palomares-Ruiz, A. (2011). El modelo docente universitario y el uso de nuevas metodologías en la enseñanza, aprendizaje y evaluación. Revista de Educación, 355, 591-604.

Scriven, M. (1967). The methodology of evaluation. In R. W. Tyler, R. M. Gagne, \& M. Scriven (Eds.), Perspectives of curriculum evaluation (pp. 39-83). Chicago: RandMcNally. 